\title{
INTERACCIÓN EN NIÑOS CON TRASTORNO DEL ESPECTRO AUTISTA
}

\section{INTERACTION IN CHILDREN WITH AUTISM SPECTRUM DISORDER}

Recibido 25 de febrero 2015 Aceptado 26 de febrero 2015

Correspondencia: Manantial de San Cristobal S/N $2^{\circ}$ piso Xalapa 2000. CP 91097 Xalapa, Ver. México Tel: 012288421700 Ext. 19518 qfb_cvb@yahoo.com.mx

\section{Autores:}

Vela Baizabal Carmelita

Universidad Veracruzana, Doctorado en Ciencias

Biomédicas

Granados Ramos Dora Elizabeth

Universidad Veracruzana, Facultad de Psicología-Xalapa, Laboratorio de Psicobiología

Palabras clave: Autismo, interacción padre-niño, comportamiento

Key Words: Autism, parent-child interaction, behavior 


\section{RESUMEN}

Actualmente existen investigaciones enfocadas en demostrar que la interacción es importante para favorecer el desarrollo cognoscitivo, del lenguaje, social y emocional de niños con desarrollo normal y de niños con Trastorno del Espectro Autista (TEA). Se ha propuesto que los padres de niños con TEA pueden actuar como coterapeutas de sus hijos en las interacciones cotidianas. El objetivo del estudio fue describir las características de comportamiento de niños con TEA al interactuar con su cuidador principal a través de juego libre. Participaron 16 niños con TEA de 4 a 12 años de edad con su cuidador principal. Se videograbó la interacción durante 15 minutos. A los padres se les indicó que establecieran un juego libre con sus hijos utilizando los materiales didácticos colocados en una caja. Se observó la videograbación para analizar la interacción considerando: actividad, movimientos estereotipados, contacto visual, respuesta impositiva o no impositiva, verbal, objetal, gestual, gestual-verbal o visual. Durante la interacción los niños estuvieron activos 80\%. El 43\% respondió en forma verbal, 35\% a través de objetos y 22\% utilizando gestos. Sólo en el 57\% de la interacción los niños establecieron contacto visual. El estudio de la interacción en niños con TEA permite analizar las dificultades que presentan en lenguaje, interacción social y desarrollo cognoscitivo. La evaluación del desarrollo mediante pruebas estandarizadas en estos niños es compleja, por lo que el análisis de las interacciones posibilita describir el desarrollo y diseñar estrategias de apoyo para que los padres favorezcan el progreso del niño en la vida diaria.

Palabras clave: autismo, interacción padre-niño, comportamiento

\section{ABSTRACT}

Currently there is research focused on demonstrating that of interaction is important for the stimulation of cognitive, social, emotional and language development of typically developing children and children with Autism Spectrum Disorder (ASD). It has been proposed that the parents of children with ASD may act as co-therapists for their children in everyday interactions. The aim of this study was to describe the behavioral characteristics of children with ASD when than interact with their primary caregiver through free play. Participated 16 children with ASD from 4-12 years old with their primary caregiver. Interaction was videotaped for 15 minutes. Parents were then instructed to establish a free play with their children using teaching materials placed in a box. Interaction was analyzed by watching the video recording, considering: activity, stereotyped movements, eye contact, impositive response or not impositive response and whether interaction was verbal, objetal, gestural, verbal or gestural-visual. During the interaction the children were active 80\%. 43\% responded verbally, 35\% using objects and 22\% through gestures. Children in only 57\% of the interaction established visual contact. The study of interaction in children with ASD to analysis of the difficulties presented in language, social interaction and cognitive development. Evaluation of developmental by standardized tests in these children is complex, so that the analysis of interactions permits the description of development and the design of support strategies for parents in order to encourage their child's progress in daily life.

Keywords: autism, parent-child interaction, behavior

\section{8}




\section{Planteamiento del problema}

Diversos investigadores proponen que las características de interacción de los padres se relacionan con el desarrollo de lenguaje, cognoscitivo y social de los niños. Estudios realizados desde 1970 han mostrado que la capacidad de respuesta, de dirección para lograr un fin y para divertirse durante la interacción, está relacionada con el desarrollo del niño?

La capacidad de respuesta de los padres influye positivamente en el desarrollo cognoscitivo y socio-emocional de niños con desarrollo normal y de niños con factores de riesgo². Esta área se ha explorado poco en niños mexicanos con TEA y de etapa escolar, por lo que se puede contribuir al desarrollo de estrategias de intervención.

Actualmente se ha propuesto que los padres de niños con TEA pueden fungir como coterapeutas de sus hijos en las interacciones cotidianas3. Por lo que en el presente estudio pretendemos describir las características de comportamiento con el análisis de la interacción entre niños con TEA y sus padres, a través de actividades de juego libre con materiales didácticos.

\section{INTRODUCCIÓN}

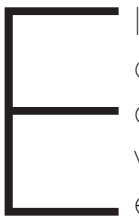

I autismo es un trastorno del neurodesarrollo caracterizado por un déficit en las habilidades sociales, en la comunicación tanto verbal como no verbal, por presencia de estereotipias e inflexibilidad en el comportamiento ante cambio de rutinas". La sintomatología del Trastorno del Espectro Autista (TEA) puede detectarse antes de los 3 años de edad, sin embargo hasta el momento no existe un biomarcador que permita confirmar el diagnóstico, por lo que su evaluación se realiza mediante observación clínica ${ }^{5}$. Se estima que afecta a 1 de cada 100 niños y su prevalencia es mayor en población masculina por cada niña con TEA hay cuatro niños. En México no existen cifras precisas, aunque el Instituto Nacional de Estadística y Geografía (INEGI) reportó en 2004, alrededor de 45000 niños con TEA.

La interacción niño-cuidador, quien regularmente es la madre del niño, en las etapas tempranas de vida ha demostrado relación con el desarrollo general del niñó. Desde el nacimiento, se establecen las pautas de organización del comportamiento y la respuesta selectiva a los estímulos sociales. Asimismo, la interacción familiar promueve un mayor desarrollo de los niños ${ }^{8}$

Diversos estudios han investigado las interacciones entre hijos y madres, incluyendo niños con repertorios conductuales limitados, con síndrome de Down, con retraso en el desarrollo, prematuros y con autismo, en los cuales se han observado que las madres tienen mayor perseverancia para que los niños puedan resolver tareas complejas individuales comparadas con madres de niños con desarrollo normal $\left.\right|^{9,10}$.

La conducta de los niños con TEA ha sido estudiada desde los años ochenta mediante la observación durante juego libre con su cuidador principal ${ }^{17}$. Se ha observado que los niños con TEA establecen varias acciones con los objetos e incluso algunos son capaces de realizar actos de juego funcional y simbólico, sin embargo el juego es diferente del observado en niños normales y en niños con retraso mental de nivel de desarrollo cognoscitivo 
semejante ${ }^{\text {2. }}$ También se ha observado que las madres de niños con trastornos del lenguaje son más sensibles a las conductas de sus hijos, comparado con las madres de niños con discapacidad intelectual y de niños con autismo ${ }^{13}$.

En México pocos estudios han explorado la conducta recíproca entre niños con TEA y su cuidador principal $^{14}$. Actualmente existen investigaciones enfocadas a demostrar que la interacción es importante para favorecer el desarrollo cognoscitivo, el lenguaje, la interacción social y emocional de niños con desarrollo normal y de niños con TEA. Se ha propuesto que los padres de niños con TEA pueden fungir como coterapeutas de sus hijos en las interacciones cotidianas ${ }^{3}$. El objetivo del estudio fue describir las características de comportamiento de niños con TEA al interactuar con su cuidador principal a través de juego libre.

\section{MATERIAL Y MÉTODOS}

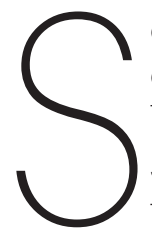

e realizó un estudio descriptivo y observacional en el que participaron 16 niños con TEA de 4 a 12 años ( 15 niños y 1 niña) con su cuidador principal. Diez niños fueron referidos del Centro Estatal para la Detección y Atención del Autismo de la ciudad de Xalapa, Veracruz y los cinco niños restantes fueron referidos de sus centros educativos. Se videograbó la interacción de cada niño con TEA y su cuidador, en situación de juego libre durante 15 minutos. A los padres se les indicó que jugaran con sus hijos utilizando materiales didácticos colocados en una caja, se les dio la siguiente indicación: "Vamos a grabar 15 minutos de juego libre con su hijo, le pedimos que juegue con él como usted lo hace en casa, utilizando los materiales que hay en la caja. Esto nos permitirá conocer cómo se comunica con su hijo y conocer el desarrollo del niño".
La interacción niño-cuidador principal se videograbó en un cubículo del Laboratorio de Psicobiología de la Facultad de Psicología de la Universidad Veracruzana. Las condiciones de observación fueron semejantes para todas las díadas, con iluminación adecuada, dos sillas y una mesa para facilitar el uso de los materiales: caja con: rompecabezas, pelotas, juguetes para agitar, rodar, dar cuerda, memoramas y muñecos de diferentes formas y texturas sin presencia de observadores.

La videograbación se analizó mediante ciclos, considerando un ciclo como el cambio en el estilo de comportamiento de inicio, respuesta o pasividad de alguno de los participantes.

Cada ciclo se clasificó con seis categorías adaptadas de Muñoz-Ledo?:

1. Activo: se observa intencionalidad de establecer intercambio o afectar la conducta del otro.

2. Pasivo: no se observa intencionalidad de establecer intercambio o afectar la conducta del otro.

3. Movimientos estereotipados: movimientos o conductas repetitivas del niño durante la interacción como aleteo de manos, balanceo de cuerpo, movimiento de piernas, etc.

4. Sin contacto visual: Cuando no se miran a la cara el niño y el cuidador.

5. Impositivo: iniciar y dirigir la atención del otro e inclusive forzarlo para realizar alguna actividad.

6. No impositivo: guiar la interacción para atraer la atención del otro con o sin objetos y ampliar el tiempo de interacción.

Los tipos de respuesta se clasificaron en tres grupos, adaptadas de Muñoz-Ledo?:

\section{0}

uMN Mhum vol.74. No. 1 enero-abril 2015 
1. Verbal: describir, comentar o responder en forma oral a una acción.

2. Objetal: se utiliza un juguete o un objeto para llamar la atención del otro.

3. Gestual: realiza gestos o movimientos corporales sin verbalizaciones.

De los 15 min videograbados, se contó el número de ciclos a partir de los 2 minutos con 30 segundos hasta los 12 minutos con 30 segundos. Posteriormente se analizaron los ciclos, se clasificaron en las categorías y tipos de respuesta.

De acuerdo a las características de los niños con TEA, se formaron cuatro grupos (G1 al G4) con cuatro participantes cada uno, donde los niños necesitaban apoyo:

G1: en el área de comunicación social o de conductas estereotipadas y no presentaban dificultades de lenguaje.

G2: en comunicación social y presentaban conductas estereotipadas. Podían presentar dificultades de lenguaje.
G3: frecuente en comunicación social y conductas estereotipadas. Presentaban lenguaje con ecolalia y dificultades de articulación.

G4: muy frecuente en todas las áreas y presentaban conductas estereotipadas que interferían con las actividades cotidianas. Había ausencia de lenguaje.

\section{RESULTADOS}

a edad promedio de los niños fue de 9 años 7 meses (D.E. \pm 31 meses). De los 16 niños, ocho presentaban ecolalia y ocho tenían lenguaje normal.

Se describirán las características de la interacción de los 16 niños con TEA y posteriormente por grupos separados según el nivel de apoyo requerido.

Todos los niños permanecieron activos en el 80\% de la interacción, respondieron con actitud impositiva el 10\%, en forma verbal el 43\%, a través de objetos el 35\% y utilizando gestos el 22\%. Los niños establecieron contacto visual sólo en el 57\% de la interacción, y en el 25\% presentaron movimientos estereotipados (Figura 1)

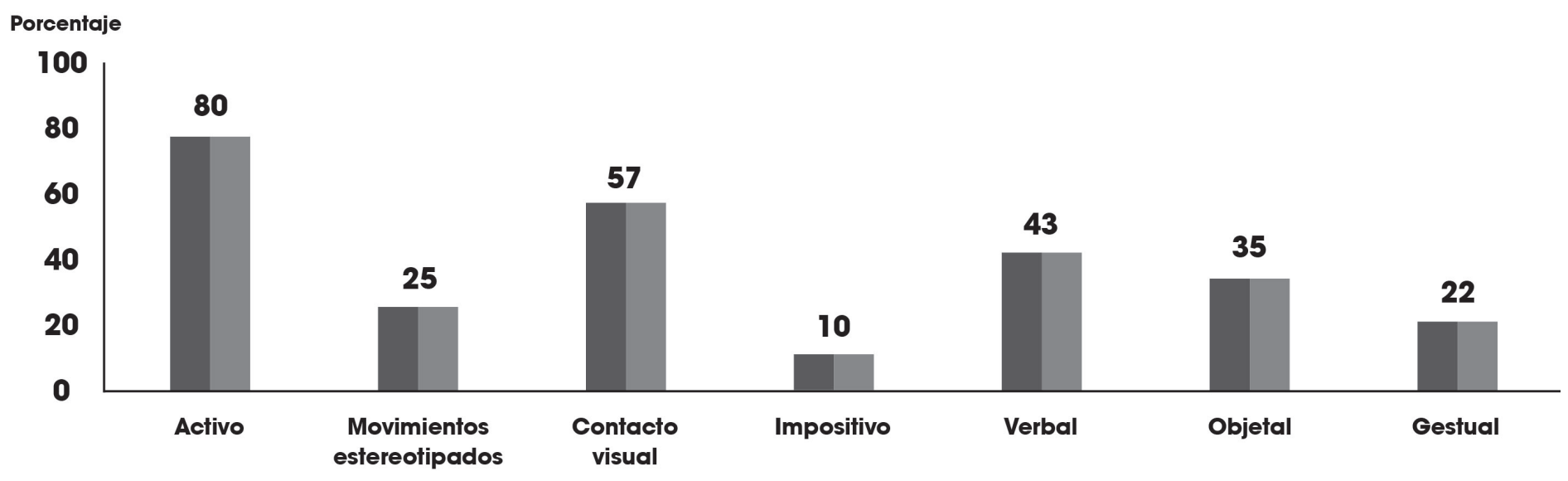

Figura 1. Porcentaje de las categorías analizadas durante la interacción de los niños con TEA. 
Los niños más activos fueron del G1 (95\%) y G3 (96\%), el G4 permaneció activo el 50\% de la interacción. Los niños que mostraron un comportamiento impositivo mayor fueron G3 y G4 (14\%). Se observaron movimientos estereotipados en todos los grupos en más del 15\%, los cuales incluían golpeteo ligero sobre la mesa, aleteo de manos, balanceo de cuerpo y movimiento de piernas. Mientras que los grupos que establecieron mayor contacto visual con su cuidador fueron G1 y G3 (Figura 2)

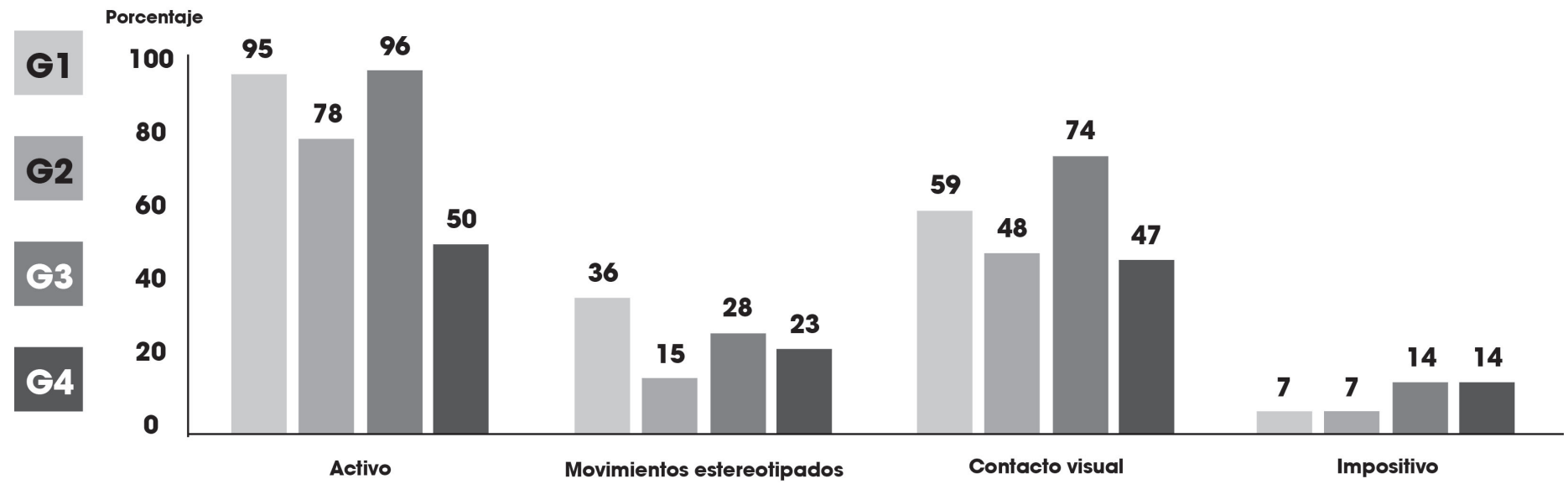

Figura 2. Porcentaje de las categorías presentes durante la interacción de los niños con TEA por grupo de estudio.

Las respuestas que predominaron en los 16 niños con TEA fueron las verbales con 43\% y objetales con 35\%. El uso de gestos sólo se observó en el 22\% de la interacción.

En la interacción, G1 y G2 expresaron en 46\% respuestas verbales y G3 en 44\%, mientras que en G4 se observó el 34\%. El uso de respuestas objetales se presentó en G4 el 46\%. Las respuestas gestuales se presentaron en G1 y G3 el 24\%, en G2 el 19\% y en G4 el 20\% (Figura 3).

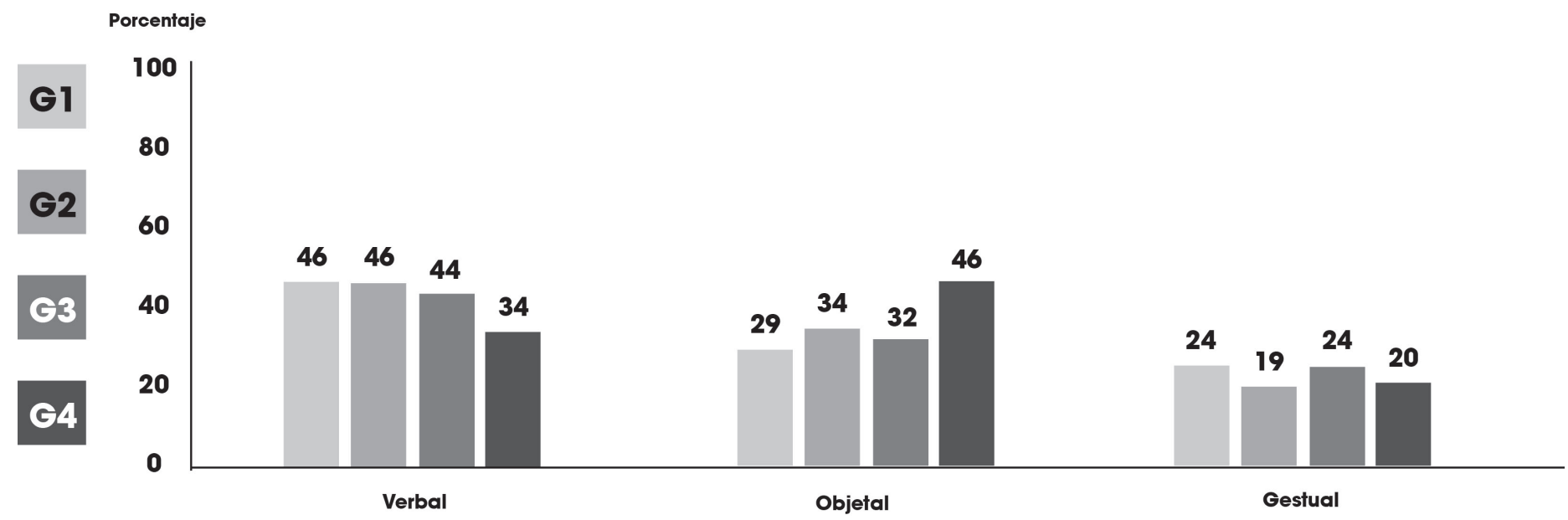

Figura 3. Porcentaje de las respuestas observadas durante la interacción de los niños con TEA divididos por grupo de estudio 


\section{DISCUSIÓN}

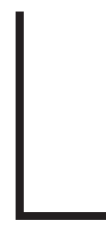

a evaluación del desarrollo y el diagnóstico mediante pruebas estandarizadas en los niños con TEA en nuestro país es compleja, ya que la mayoría de los estándares de oro que se utilizan no están validados para población mexicana ${ }^{15}$, sin embargo el análisis de las interacciones en situaciones cotidianas permite describir el desarrollo de los niños y establecer las bases para diseñar estrategias de apoyo en casa, para que los padres favorezcan el progreso del niño.

Se cumplió el objetivo de describir las características de comportamiento de niños con TEA al interactuar con su cuidador principal a través de juego libre. Se observaron las respuestas verbales, objetales y gestuales, así como los movimientos estereotipados durante las actividades de juego, y el establecimiento de contacto visual.

Los niños con TEA evaluados estuvieron activos el 80\% de la interacción, lo cual fue similar a los niños en edad preescolar con coeficiente intelectual promedio descritos por Eheart, quien analizó que en la interacción respondían el 78\%, mientras que los niños con coeficiente intelectual bajo lo hicieron sólo en el 56\% del tiempo ${ }^{10}$.

Aunque la actividad en la interacción de los niños con TEA fue semejante a los niños sin dificultades, se deben analizar los tipos de respuestas en los diferentes grupos de estudio, como el lenguaje, uso de objetos y gestos para caracterizar su comportamiento en forma detallada. En los casos presentados, se observó que a mayor apoyo, hubo menor uso de gestos y mayor uso de objetos, mientras que a menor apoyo del TEA se observaron mayor uso de respuestas verbales y gestos.

Los estudios demuestran que el análisis de los gestos en niños con TEA puede ayudar a identificar diferentes fenotipos en el autismo y a desarrollar nuevas terapias ${ }^{16}$.

En la interacción de los niños con TEA y sus padres, el $43 \%$ del tiempo utilizaron lenguaje verbal, lo cual coincide con el análisis realizado por Doussard et al., quienes describen más contacto físico de las madres hacia los niños con TEA, y menos respuestas verbales, comparado con las madres de niños sin dificultades de desarrollo ${ }^{17}$.

Diversos autores proponen que las características de la interacción de los padres se relacionan con el desarrollo cognoscitivo 2, 16. En el presente estudio se observó que a mayores dificultades en los niños, los padres fueron impositivos, esto puede ser desfavorable, ya que una interacción sin dirección no contribuye al desarrollo de los niños ${ }^{18}$

Es importante que las estrategias de apoyo estén dirigidas al uso de lenguaje para favorecer la comunicación en los niños con TEA y al desarrollo social en la interacción con los padres y las personas que convivan en casa y en su entorno.

\section{CONCLUSIONES}

I os niños con TEA que más apoyo requerían utilizaban menos gestos e interactuaban a través de objetos con sus padres. Los niños con TEA que necesitaron menos apoyo de

L_ sus padres, utilizaron más respuestas verbales y gestos en la interacción.

En los programas de apoyo dirigidos a la atención de niños escolares con TEA deben incluirse estrategias que favorezcan la interacción con calidad a partir del contacto visual, desarrollo del lenguaje y habilidades cognoscitivas a través de la guía no impositiva.

\section{Agradecimientos:}

A los niños, padres y personal del Centro Estatal para la Detección y Atención del Autismo del Estado de Veracruz.

Al Consejo Nacional de Ciencia y Tecnología por la beca 281550 del Doctorado en Ciencias Biomédicas de la Universidad Veracruzana. 


\section{REFERENCIAS BIBLIOGRÁFICAS}

1. Kochanska $G$, Forman $G$ y Coy KC. Implications of the mother-child relationship in infancy for socialization in the second year of life. Infant Behav Dev. 1999; 22:249-265.

2. Moore JB, Saylor CFy Boyce GC. Parent-child interaction and developmental outcomes in medically fragile, high risk children. Children „s Health Care. 1998; 27(2):97-112.

3. Ruble L, Dufle A, King A y Lorenz D. Caregiver responsiveness and social interaction behaviors of young children with autism. Topics Early Child Spec Educ. 2008; 28: 158-170.

4. American Psychiatric Association. Diagnostic and statistical manual of mental disorders 5. Washington DC: American Psychiatric Association Press. 2013; 50-59

5. Fombonne E. Epidemiological surveys of autism and other pervasive developmental disorders: an update. J Autism Dev Disord. 2003; 33(4):365-382.

6. Oliviera A, Granados D, Romero G, Alcaraz V, Martínez R, Uresti L y Gálvez V. (Mayo, 2008). Interacción madre-hijo y desarrollo en preescolares con dillcultades de lenguaje. $V$ encuentro. Participación de la mujer en la ciencia. Centro de Investigaciones en óptica. León, Guanajuato, México.

7. Muñoz-Ledo P, Cravioło J, Méndez I, Sánchez M y Mandujano M. Interacciones madre-hijo y desarrollo infantil: aportes teóricos y metodológicos de estudio en niños con antecedentes de daño neurológico perinatal. UAM. 2003; 7-130.

8. Guralnick MJ. A developmental systems model for early intervention. Infants Young Child. 2001; 14(2): 1-18.

9. Cielinski K, Vaugh BE, Seifer R y Contreras J. Relations among sustained engagement during play, quality of play, and mother-child interaction in samples of children with Down syndrome and normally developing toddlers. Infant Behav Dev. 1995; 18:163-176.

10. Eheart BK. Mother-child interactions with non-retarded and mentally retarded preschoolers. Am J Ment Del̈c. 1982; 87:20-25.

11. Baron-Cohen S. Autism and symbolic play. Br J Dev Psychol. 1987; 5: 139-148.

12. Canal R y Rivieré $A$. Conducta de juego y expresiones emocionales de niños autistas no verbales en una situación natural de interacción. La esperanza no es un sueño. Actas del V Congreso Internacional Autismo-Europa. Fundación ONCE. Madrid. 1998; 475-492.

13. Díken $॥$. An exploration of interactional behaviors of turkish mothers and their children with special needs: implications for early intervention practices. Education and Science. 2012; 37(163):297-309.

14. Damián M. Estilos de interacción de una paraprofesional y la madre de una niña autista. Psicothema. 2000; 12(2): 166-169.

15. Bravo A, Esmer C y Navarro ME. Autism Spectrum Disorders in Mexico. Comprehensive Guide to Autism. New York:Springer; 2014; pp. 2469-2482.

16. Mastrogiuseppe $M$, Capirci $O$, Cuva S y Venuti P. Gestural communication in children with autism spectrum disorders during mother-child interaction. Autism. 2014;1-13.

17. Doussard-Roosevelt JA, Joe C, Bazhenova OV y Porges SW. Mother-child interaction in autistic and nonautistic children: characteristics of maternal approach behaviors and child social responses. Dev Psychopathol. 2003; 15:277-295.

18. Mahoney $G$ y Powell A. Modifyng parent-child interaction: enhancing the development of handicapped children. J Spec Educ. 1988; 22:82-96. 